\title{
Aerosol Mediated Transmission of SARS-Cov-2 Or COVID-19 In the Cardiac Surgical Operating Room
}

\author{
Satish Kumar ${ }^{1}$, Lalit Kapoor ${ }^{1}$, dhiraj barman ${ }^{2}$, and Pradeep Narayan ${ }^{1}$ \\ ${ }^{1}$ Rabindranath Tagore International Institute of Cardiac Sciences \\ ${ }^{2}$ Affiliation not available
}

May 20, 2020

\begin{abstract}
While there is significant awareness regarding droplet and contact transmission, aerosols are generally underestimated as a potential mode of transmission of SARS-Cov-2 infection. With the gradual resumption of cardiac surgical activities, the cardiac surgical operating room will become an important potential source of infection to the cardiac surgeon and other healthcare workers participating in the operation. There is also diminished awareness about the different aerosol generating procedures (AGP) in the cardiac surgical operating room. In this mini-review we intend to highlight the various aerosol generating procedures that are common in cardiac surgery. This will help increase the awareness among surgeons to AGP. A practical approach to taking preventive measures have also been discussed.
\end{abstract}

\begin{abstract}
While there is significant awareness regarding droplet and contact transmission, aerosols are generally underestimated as a potential mode of transmission of SARS-Cov-2 infection. With the gradual resumption of cardiac surgical activities, the cardiac surgical operating room will become an important potential source of infection to the cardiac surgeon and other healthcare workers participating in the operation. There is also diminished awareness about the different aerosol generating procedures (AGP) in the cardiac surgical operating room. In this mini-review we intend to highlight the various aerosol generating procedures that are common in cardiac surgery. This will help increase the awareness among surgeons to AGP. A practical approach to taking preventive measures have also been discussed.

Apart from droplet infection and transmission through fomites it is now well established that coronavirus can spread through aerosols as well.(1) However, a significant research gap exists in the epidemiology of the risk of transmission of infections from patients undergoing aerosol generating procedures (AGP). (2) AGP are generally thought to be a concern for the anaesthetists and the risk to cardiac surgeons is grossly underestimated.

Even before the current corona pandemic, a global outbreak of invasive Mycobacterium chimaera has been reported in cardiac surgery due to aerosol release. This occurred through breaches in the heater-cooler units used in cardiopulmonary bypass circuits. (3) Thus, the risk of aerosol mediated transmission is very real and the need for safety measures are extremely practical.

The COVID-19 virus spreads predominantly through droplet and aerosol routes and blood-borne infection is not considered a major source of transmission .(4) There are some major differences between droplet and airborne transmission that leads to airborne transmission in the operating room more of a hazard than the droplet route.
\end{abstract}


Based on e electron microscopy the size of the coronavirus-shaped spherical particles is estimated to be about 0.125 microns $(125 \mathrm{~nm})$ and ranges from 0.06 microns to 0.14 microns. (5) While droplet infections are via larger respiratory particles, generally above $5 \mu \mathrm{m}$ diameter, and are subject to gravitational forces, aerosol mediated transmission occurs with smaller respiratory particles (generally $<5 \mu \mathrm{m}$ ) circulating in the air. As a result, while contact is necessary for droplet infections and thereby handwashing and gloves are highly effective against contact transmission, viral particles transmitted though aerosol is absorbed via the respiratory mucosa and potentially across the conjunctivae other measures are required to prevent transmission. These smaller viral particles $(<10 \mu \mathrm{m})$ are most likely to penetrate deeply into the lung and cause infection.(6)The radius of spread is also different and is no more than one meter for droplet infections. However, because of the smaller size the SARS-CoV-2 or COVID-19 as it is popularly known as, can spread across larger areas and has been shown to remain viable in aerosols even at 3 hours.(7)

The highest viral load of the virus causing COVID-19, is in sputum and upper airway secretions and endotracheal intubation is the commonest and most relevant aerosol generating procedure in cardiac surgery. (8)

Apart from intubation and extubation, bag mask ventilation, suctioning of airways, insertion of chest drains and thoracotomies can all lead to aerosol generation. (9) In paediatric cardiac surgery, valve repairs and at times in coronary artery bypass operations as well, transesophageal echocardiography (TEE) is often used. TEE carries an increased risk of transmission of SARS-CoV-2. While this risk is greater in non-intubated patients, viral transmission may still occur through direct contact with the patient's secretions, resulting in contaminated hands and surfaces with the potential to infect not just the echocardiographers but also other personnel in the operating room. (10) Sternotomy requires a high -speed device and is considered to be a procedure that leads to blood and tissue fluid aerosolization. (4) Surgical smoke produced by heat generating devices in cardiac surgery can also contain chemicals, blood and tissue particles, bacteria, and viruses. (11)

Carbon di-oxide (CO2) insufflation in the operative field is practiced in both open cardiac surgical procedures as well as minimally invasive cardiac surgery to aid in de-airing and prevention of air embolism. This has more relevance in minimally invasive cardiac surgery as minimal invasive cardiac surgery often does not permit normal de-airing maneuvers. (12) OPCAB surgery relies heavily on using a blower-mister which used $\mathrm{CO} 2$ with saline and perhaps contribute to aerosol generation as well.(13)Disconnection of ventilatory circuits during use; cardiopulmonary resuscitation (before tracheal intubation); bronchoscopy; and tracheal suction without a 'closed in-line system, nasogastric tube insertion are the other examples of aerosol generating procedures. (8)

Prevention of aerosol-based transmission requires two approaches. Firstly, minimizing aerosol generating procedures and secondly protecting against exposure.

Minimizing aerosol generation:

Since the greatest risk of aerosol generation is from endotracheal intubation preventive measures should primarily focus on minimizing the risk at this very stage. It is recommended that adequately ventilated single rooms should be used when performing aerosol-generating procedures. Hospitals could make adjustments to their intubation and operating practices. Ideally it should be seen if it is possible to convert any of the operating rooms to negative pressure environments with airflow changes ( $>12$ air flow changes/hour).(8) Failing this, patients could be intubated in a room next to the operating room. If such a room does not exist and if there are two OR's next to each other one of them could perhaps be used as an anesthetic room for intubating and the other one for operating.

However, most of the centers across the world, the intubation is performed is either performed in a positive pressure environment or often even inside the operating room. The positive pressure airflow environment of the operating room is a risk factor for viral spread and thus aerosols once released actually stay in the environment for 3 hours or longer which often is the duration of the cardiac surgical procedure. In these situations, following intubation of the patient, the air conditioning should be turned off and after 20 minutes to allow for the droplets and aerosols to settle, the floors should be cleaned. This is known to clear the 
settled aerosols without refreshing the spread.(8)

Reducing the use of blower-mister during OPCAB as far as practicable and depending on traditional measures for de-airing after open cardiac surgery in preference to CO2 insufflation would also be useful in reducing the aerosol load. So, TEE should only be performed in intubated patients only in those perioperative situations in which the benefits outweigh the risks.(10)

Protecting against exposure:

The minimum number of personnel that can safely conduct the procedure should be present in the OR. Insertion of intra-pleural chest drains at the start of the procedure and connecting them to the wall suction may reduce the aerosol generated from the electro-cautery as well as from the blower-mister in OPCAB surgery.

There is some evidence that double gloving for tracheal intubation might provide extra-protection and minimize spread by fomite contamination of equipment and surroundings.(10)

The use of fluid-repellent long sleeved gown, eye protection, respirators and gloves are recommended to protect against aerosol mediated transmission of infection. Eye protection should include protection from side exposure with side shields or goggles.

The most common types of respirators in healthcare are N95 filtering facepiece respirators (FFRs), surgical N95 FFRs, and powered air purifying respirators (PAPRs). PAPRs reduce the aerosol concentration inhaled by the wearer to at least $1 / 25$ th of that in the air, compared to a $1 / 10$ th reduction for FFRs. PAPRs provide increased protection and decrease the likelihood of infection transmission to the wearer as compared to FFRs however there a PAPR has limited downward vertical field of view as well as because of the blower noise may present difficulty in communicating.(14) Evidence from systematic review and meta-analysis have failed to confirm the superiority of one type of mask (FFP3/FFP2/N95) over another (surgical facemask).(15) This could be partly because of issues with doffing off techniques of personal protective equipment and other training issues.

Conclusions: Awareness about aerosol generating procedures is key to prevent transmission of aerosol mediated SARS-Cov-2 infection. Minimizing aerosol generation as far as practicable and taking measures to prevent exposure are important for ensuring safety of cardiac surgeons and other team members.

References:

1. Fathizadeh H, Maroufi P, Momen-Heravi M, Dao S, Köse Ş, Ganbarov K, et al. Protection and disinfection policies against SARS-CoV-2 (COVID-19). Infez Med. 2020 Jun 1;28(2):185-91.

2. Tran K, Cimon K, Severn M, Pessoa-Silva CL, Conly J. Aerosol Generating Procedures and Risk of Transmission of Acute Respiratory Infections to Healthcare Workers: A Systematic Review. PLoS ONE [Internet]. 2012 Apr 26 [cited 2020 May 18];7(4). Available from: https://www.ncbi.nlm.nih.gov/pmc/articles/PMC3338532/

3. Diekema DJ. MYCOBACTERIUM CHIMAERA INFECTIONS AFTER CARDIOVASCULAR SURGERY: LESSONS FROM A GLOBAL OUTBREAK. Trans Am Clin Climatol Assoc. 2019;130:136-44.

4. Cook TM. Personal protective equipment during the coronavirus disease (COVID) 2019 pandemic - a narrative review. Anaesthesia. 2020 Apr 4;

5. Zhu N, Zhang D, Wang W, Li X, Yang B, Song J, et al. A Novel Coronavirus from Patients with Pneumonia in China, 2019. N Engl J Med. 2020 20;382(8):727-33.

6. Gralton J, Tovey E, McLaws M-L, Rawlinson WD. The role of particle size in aerosolised pathogen transmission: a review. J Infect. 2011 Jan;62(1):1-13.

7. van Doremalen N, Bushmaker T, Morris DH, Holbrook MG, Gamble A, Williamson BN, et al. Aerosol and Surface Stability of SARS-CoV-2 as Compared with SARS-CoV-1. N Engl J Med. 2020 16;382(16):1564-7. 
8. Cook TM, El-Boghdadly K, McGuire B, McNarry AF, Patel A, Higgs A. Consensus guidelines for managing the airway in patients with COVID-19: Guidelines from the Difficult Airway Society, the Association of Anaesthetists the Intensive Care Society, the Faculty of Intensive Care Medicine and the Royal College of Anaesthetists. Anaesthesia. 2020;75(6):785-99.

9. Engelman DT, Lother S, George I, Funk DJ, Ailawadi G, Atluri P, et al. Adult Cardiac Surgery and the COVID-19 Pandemic: Aggressive Infection Mitigation Strategies are Necessary in the Operating Room and Surgical Recovery. Ann Thorac Surg. 2020 Apr 27;

10. Nicoara A, Maldonado Y, Kort S, Swaminathan M, Mackensen GB. Specific Considerations for the Protection of Patients and Echocardiography Service Providers When Performing Perioperative or Periprocedural Transesophageal Echocardiography During the 2019 Novel Coronavirus Outbreak: Council on Perioperative Echocardiography Supplement to the Statement of the American Society of Echocardiography. J Am Soc Echocardiogr. 2020 Apr;S0894731720302194.

11. Liu Y, Song Y, Hu X, Yan L, Zhu X. Awareness of surgical smoke hazards and enhancement of surgical smoke prevention among the gynecologists. J Cancer. 2019;10(12):2788-99.

12. Nyman J, Svenarud P, Linden J van der. Carbon dioxide de-airing in minimal invasive cardiac surgery, a new effective device. J Cardiothorac Surg. 2019 Dec;14(1):1-8.

13. Plass CA, Podesser BK, Prusa AM. Effect of blower-mister devices on vasoreactivity of coronary artery bypass grafts. J Thorac Cardiovasc Surg. 2010 Oct 1;140(4):923-7.

14. CDC. Coronavirus Disease 2019 (COVID-19) [Internet]. Centers for Disease Control and Prevention. 2020 [cited 2020 May 18]. Available from: https://www.cdc.gov/coronavirus/2019-ncov/hcp/ppestrategy/powered-air-purifying-respirators-strategy.html

15. Effectiveness of Masks and Respirators Against Respiratory Infections in Healthcare Workers: A Systematic Review and Meta-Analysis. - PubMed - NCBI [Internet]. [cited 2020 May 18]. Available from: https://www.ncbi.nlm.nih.gov/pubmed/29140516 